\title{
P I 4-08. Intramuscular DNA delivery by electroporation leads to greatly enhanced systemic and mucosal immune responses and control of SIVmac25 I challenge
}

\author{
GN Pavlakis*1, M Rosati ${ }^{1}$, V Patel ${ }^{1}$, A Valentin ${ }^{1}$, C Bergamaschi ${ }^{1}$, V Kulkarni ${ }^{2}$, \\ R Jalah ${ }^{2}$, A von Gegerfelt ${ }^{1}$, C Ohlen ${ }^{3}$, A Khan ${ }^{4}$, R Draghia-Akli ${ }^{4}$, K Van \\ Rompay ${ }^{5}$ and BK Felber ${ }^{2}$
}

Address: ${ }^{1}$ Center for Cancer Research, HRS, VB, NCI-Frederick, Frederick, USA, ${ }^{2}$ HRPS, VB, NCI-Frederick, Frederick, USA, ${ }^{3}$ ACVP, SAIC-Frederick, Inc., NCI at Frederick, Frederick, USA, ${ }^{4}$ VGX Pharmaceuticals, Inc., The Woodlands, USA and ${ }^{5}$ California National Regional Primate Research Center, Davis, CA, USA

* Corresponding author

from AIDS Vaccine 2009

Paris, France. 19-22 October 2009

Published: 22 October 2009

Retrovirology 2009, 6(Suppl 3):PI 96 doi:10.1 186/1742-4690-6-S3-PI96

This abstract is available from: http://www.retrovirology.com/content/6/S3/PI96

(c) 2009 Pavlakis et al; licensee BioMed Central Ltd.

\section{Background}

DNA vaccination is a promising technology, but the reported immunogenicity in human trials is low. Here we use optimized DNA vectors and intramuscular electroporation procedures to show great increases in immunogenicity of DNA vaccines and protection from high viremia after challenge.

\section{Methods}

Indian rhesus macaques were vaccinated intramuscularly by EP with optimized DNA vectors producing the majority of SIVmac239 proteins. Immune responses were monitored by flow cytometry. Animals were challenged rectally with high dose SIVmac521 and plasma viral loads and immune responses were monitored.

\section{Results}

DNA delivery using EP led to greatly enhanced expression of SIV antigens and increased cellular responses (up 3\% of total T cells) that were broad, long-lasting (up to 45 weeks post vaccination) and included cells of multifunctional phenotype. SIV-specific cellular responses were found both in blood, BAL and rectal mucosa. Interestingly, compared to blood, the responses in BAL were consistently higher (up to $2 \log$ ) and included a higher frequency of polyfunctional cells. The cellular responses were characterized as predominantly EM $\mathrm{CD} 4^{+}$and $\mathrm{CD} 8^{+} \mathrm{T}$ cells in BAL and as both CM and EM T cells in blood. EP DNA delivery elicited both systemic and mucosal humoral immune responses, including the induction of Gag-specific IgA. Upon high dose SIVmac251 challenge, the vaccinated animals showed statistically significant lower VL peak in acute $(1 \log 10)$ and in chronic viremia (1.7 $\log 10)$.

\section{Conclusion}

The combination of optimized DNA vectors and improved delivery by EP led to great induction of SIV-specific systemic and mucosal immune responses. DNA vaccination in the absence of any heterologous boost can provide protection from high viremia comparable to any other vaccine modality tested in this macaque model. The immunological and virological benefit achieved in the macaque model provides critical information to improve DNA vaccination efficacy in humans. 